Meta

Journal des tradlucteurs

Translators' Journal

\title{
Or/gold : étude comparative
}

\section{Suzanne Grenier-Larocque}

Volume 26, numéro 2, juin 1981

URI : https://id.erudit.org/iderudit/002969ar

DOI : https://doi.org/10.7202/002969ar

Aller au sommaire du numéro

Éditeur(s)

Les Presses de l'Université de Montréal

ISSN

0026-0452 (imprimé)

1492-1421 (numérique)

Découvrir la revue

Citer cet article

Grenier-Larocque, S. (1981). Or/gold : étude comparative. Meta, 26(2), 172-175.

https://doi.org/10.7202/002969ar d'utilisation que vous pouvez consulter en ligne.

https://apropos.erudit.org/fr/usagers/politique-dutilisation/ 


\section{OR/GOLD : Étude comparative.}

Les récentes recherches que j'ai faites sur le mot or et son homologue anglais gold me semblent d'actualité, car l'or fait souvent la manchette dans le contexte économique actuel.

Il est assez intéressant de noter qu'en général il y a équivalence entre les différentes expressions françaises et anglaises. Il n'est pas de mon intention de présenter ici une recherche exhaustive, mais simplement de comparer les expressions les plus connues, laissant de côté les aspects techniques et scientifiques du métal précieux.

LANGUE DE DÉPART : FRANÇAIS

La Poule aux cufs d'or

Boucles d'or

La parole est d'argent mais le silence est d'or

Tout ce qui brille n'est pas or

Le feu éprouve l'or et l'or éprouve le caractère
The Goose with the Golden Eggs.

Goldilocks

Speech is silver, silence is golden

All that glitters is not gold

Fire is the test of gold, adversity, of strong men
Conte pour enfants

Héroine de conte

Proverbe

Proverbe

Maxime 


\author{
Adorer le veau d'or \\ La Toison d'Or \\ L'Âge d'Or
}

Le Scarabée d'Or

Verge d'or

Bouton d'or

Pour tout l'or du monde

C'est de l'or en barres

Une affaire d'or

Une vraie mine d'or

Un cour d'or

Nager dans l'or, marcher sur

l'or, être cousu d'or

Livre d'or

Noce d'or

Or blanc

Or noir
To worship the golden calf

The Golden Fleece

The Golden Age

\section{The Gold Bug}

Goldenrod

Buttercup

For all the tea of China

It's as safe as the Bank of England

A golden opportunity

A regular gold-mine

A heart of gold

To be rolling in money

Visitor's book

Golden wedding

White gold

Black gold
Maxime tirée de la Bible

Mythologie

Myth. : ère de paix et de bonheur. S'emploie aussi au figuré

Litt. (de E. A. Poe)

Plante

Plante

Expr. populaire

Expr. populaire

Expression

Expression

Expr. populaire

Expr. populaire

Registre pour visiteurs de marque

$50^{e}$ anniversaire

Alliage d'or avec d'autres métaux pour lui donner lapparence du platine

Pétrole

\section{LANGUE DE DÉPART : ANGLAIS}

Gold plate does not fill your belly

To sell someone a gold brick Gold fever

Gold rush

Fool's gold

Gold flux

Gold fish

Gold filling

Gold braid

It's worth its weight in gold

Gold standard

Gold reserve

Gold Coast

The Man with the Golden Gun

Goldfinger
La belle cage ne nourrit pas l'oiseau

Escroquer quelqu'un

La fièvre de l'or

La ruée vers l'or

Pyrite

Aventurine

Poisson rouge

Aurification

Galon d'officier

Cela vaut son pesant d'or

L'étalon or

Réserve métallique or

Côte-d'Or

L'Homme au pistolet d'or Goldfinger
Maxime

Expr. populaire

Expr. rappelant la fièvre de 1849 en Californie

Expr. rappelant la ruée de 1849 en Californie

Sulfure naturel de fer qui ressemble à de lor

Variété de quartz

Chirurgie dentaire

Militaire

Expr. populaire

Finance

Finance

Département de la France

Film de James Bond

Film de James Bond

CAS DE POLYSÉMIE, D'ALLOMORPHISME,

ET CAS OU JE N'AI PAS TROUVÉ D'ÉQUIVALENCE:

Étoile d'or: - Médaille décernée aux Héros de l'Union Soviétique. (Larousse).

Gold star : - A small gold star of cloth, paper, etc. awarded as a mark of honor in school, etc., or displayed to represent a member of the armed forces killed in the line of duty in war time. (Webster).

Règle d'or: - Règle dont l'application ne peut être que profitable. 
- Précepte imposé dès le Moyen Âge au chanteur, et qui lui recommandait de ne pas respirer ni s'arrêter en passant d'une syllabe à l'autre, ou d'un mot à l'autre s'ils sont unis par le sens. (Larousse).

Golden rule : $\quad$ - The rule of three (in math.) (Oxford).

- The rule of doing to others as you would have them do toward you. (Webster).

Nombre d'or: - Un des cinq éléments du comput servant à déterminer la date de Pâques. (Larousse).

- Nombre correspondant à une proportion considérée comme particulièrement esthétique en architecture. (Larousse).

- En chimie, il existe aussi un nombre d'or, c'est le nombre d'or d'un colloïde. (Larousse).

Golden number: - A number once written in gold in calendars, indicating the year of the lunar cycle; it is used in calculating the date of Easter. (Oxford).

L'or supplée

souvent au mérite:- Wealth often makes up for lack of quality. (Traduction personnelle.)

The pot of gold

at the end of the

rainbow:

- Un trésor inaccessible, (trad. personnelle), ou encore: rêver en couleur.

\section{CONCLUSION}

La majorité de ces expressions exploitent le côté positif du terme : bonté, excellence, opulence, prospérité, etc. Quelques expressions mettent en évidence l'aspect négatif, le sentiment néfaste que l'or peut provoquer chez certains : fool's gold, gold fever, to sell someone a gold brick, etc. Un autre point assez intéressant à souligner : les dictionnaires français et anglais ne concordent pas quant à la définition à donner à certains termes : étoile d'or/gold star, règle d'or/golden rule, nombre d'or/golden number.

Suzanne Grenier-LarocQue

\section{BIBLIOGRAPHIE}

DUPRÉ, P., Encyclopédie du bon français dans l'usage contemporain, Paris, Éd. de Trévise, 1972, 3 vol.

MALOUX, Maurice, Dictionnaire des proverbes, sentences et maximes, Paris, Larousse, 1972.

DAVAU, Maurice, Marcel Cohen et Maurice Lallemand, Dictionnaire du français vivant, Montréal, Bordas, 1972.

Grand Larousse encyclopédique, Paris, Larousse, 1970, 24 tomes.

Webster's New Twentieth Century Dictionary, second edition, New York, The World Publishing Company, 1958.

The Oxford English Dictionary, Oxford, Clarendon, 1970, 12 vol. and suppl. 
Harrap's New Standard French and English Dictionary, Part One, French-English, London, Harrap, 1974, 2 vol.

Harrap's Standard French and English Dictionary, Part Two, English-French, London, Harrap, 1977. 